Running head: Peripheral attention and scalp potential

\title{
ATTENTION EFFECTS AT AUDITORY PERIPHERY DERIVED FROM HUMAN SCALP POTENTIALS: DISPLACEMENT MEASURE OF POTENTIALS
}

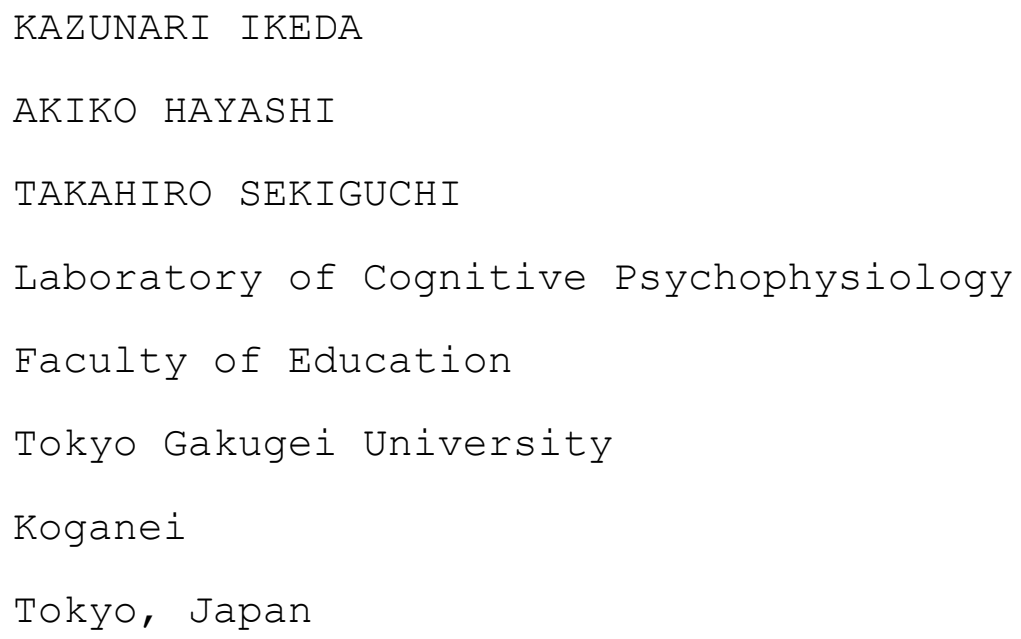




\section{Abstract}

It is known in humans that electrophysiological measures such as the auditory brainstem response (ABR) were difficult to identify the attention effect at the auditory periphery, whereas the centrifugal effect has been detected by measuring otoacoustic emissions (OAE). This research developed a measure responsive to the shift of human scalp potentials within a brief post-stimulus period (13 ms), i.e., displacement percentage, and applied it to an experiment to retrieve the peripheral attention effect. In the present experimental paradigm, tone pips were exposed to the left ear while the other ear was masked by a white noise. Twelve participants each conducted two conditions of either ignoring or attending to the tone pips. Relative to averaged scalp potentials in the ignoring condition, the shift of the potentials was found within early component range during the attentive condition, and displacement percentage then revealed a significant magnitude differencebetween the two conditions. These results suggest that, using a measure representing the potential shift itself, the peripheral effect of attention can be detected from human scalp potentials.

Keywords attention, auditory periphery, scalp potential, displacement, auditory brainstem response, olivocochlear system 
The attention effect at the auditory periphery has been a history of controversies. In their pioneering study, Hernández-Peón et al. (1956) observed in cats the reduced amplitude of click-evoked responses in the cochlear nucleus when the animals oriented to stimuli other than the auditory modality. Although this research was the first indication that the auditory function at periphery might be modulated by attention, the following studies attributed the findings to experimental artifacts (Marsh et al., 1962; Wickelgren, 1968). However, later well-controlled experiments in cats demonstrated the reduction of auditory-evoked responses at periphery (round window and cochlear nucleus) during visual tasks (Oatman, 1971, 1976; Oatman \& Anderson, 1977), clearly supporting the findings by Hernández-Peón et al. (1956). Therefore, it would be fair to say at least in cats that the attention effects modulate sensory signals at the auditory periphery. Moreover, recent researches examining an otoacoustic emission (OAE) in humans have revealed positive outcomes for such an attention effect. Thus, the OAE amplitude decreased when attention was directed to visual stimuli (Ferber-Viart et al., 1995; Froehlich et al., 1993; Meric \& Collet, 1992; Puel et al., 1988, 1989) while that amplitude augmented when auditory stimuli for evocation were paid attention (Giard et al., 1994). Because the OAE is a weak sound emitted by the cochlea spontaneously or in response to auditory stimuli, the above results would establish the peripheral attention effects in humans.

On the other hand, most of electrophysiological studies in humans have revealed negative evidences for the auditory attention effect at periphery. Among the auditory event-related potentials (ERP), an earliest attention effect can be found in the 
middle-latency range with the onset latency of $15 \mathrm{~ms}$ or later (Hackley et al., 1990; McCallum et al., 1983; Woldorff \& Hillyard, 1991). In contrast, the ERPs with the latency less than $15 \mathrm{~ms}$ were difficult to show the attention-related modulation. The whole nerve or compound action potentials (AP) in electrocochleography exhibited no difference in amplitude or latency between visual and auditory attention tasks (Kuk \& Abbas, 1989; Picton et al., 1971). In components of the auditory brainstem response (ABR), the effects of voluntary attention to or ignoring auditory stimuli were difficult to be detected (Connollyetal., 1989; Davis \& Beagley, 1985; Gregory et al., 1989; Hackley et al., 1990; Kuk \& Abbas, 1989; Picton \& Hillyard, 1974; Picton etal., 1981; Woods \& Hillyard, 1978 ) with some exceptions (Bauer \& Bayles, 1990; Brix, 1984 ; Lukas, 1980, 1981). Only a few researches measuring the frequency following response (FFR) have obtained positive results upon the existence of the peripheral attention effects (Galbraith et al., 1998; Hoormann et al., 2000).

Making a reexamination of previous studies using the ABR measurement, however, we noticed in the data some characteristic of waves that might be related to the attention effects in periphery (Ikeda etal., 2004). Thus, in the studies irrespective of positive or negative evidences for the attention effects on the ABR, the potentials within the 10-ms latency, particularly up to the latency of waveV, frequently demonstrated a task-related shift which seemed to be independent of the ABR components such as waves I-V (Brix, 1984; Connolly et al., 1989; Hirschhorn \& Michie, 1990; Lukas, 1980, 1981; Pictonetal., 1981; Woods \& Hillyard, 1978). An example of typical waveforms can be seen in Figures $2 \mathrm{a}$ and $3 \mathrm{a}$ of connolly et al. (1989). As shown in the figures, the direction of the 
potential shift seemed to be not fixed in one polarity across participants, which is in contrast with the fixed polarity for the ordinary evoked potentials. This instabilityin polaritywould be recognized in Figure 17 of Picton et al. (1981) where the early potential shift due to the tasks was particularly noteworthy for individual outcomes, while grand means over participants almost canceled out the early shift in the waveforms. Lukas (1980, 1981) proposed experimental conditions that would promote the attention effects on the ABR, and in our view, at least two of them might contribute to the occurrence of the early potential shift discussed above, i.e., low stimulus intensity and short trial lengths. Lukas (1980, 1981) utilized tone pips with intensity at $50 \mathrm{~dB}$ SL, giving rise to reduced amplitudes of waves I-III that made the task-related potential shift easy to notice. This evidence might show that the early potential deflection is a phenomenon distinguishable from typical ABR components such as waves I-V, and it survives in waveforms even though the ABR components are diminished due to low stimulus intensity. If Figures 1 and 2 in Brix (1984) are compared, moreover, the task-related displacement in the ABR waveforms is more prominent with 200 averaged trials (Figure 1) than the case averaged over 1300 trials (Figure 2). This suggests that short trial lengths, typically less than 1000 trials (Bauer \& Bayles, 1990; Brix, 1984; Lukas, 1980, 1981), might facilitate the occurrence of the early potential displacement. At a glance, the above characteristics of the early potential shift could be attributed to artifacts other than neural events in the auditory periphery, whereas we have endeavored in our studies to prove the postulation that the phenomenon reflects the task-related neural events in the auditory pathway (Ikeda et al., 2004). 
Considering the proposition raised by Lukas (1980, 1981), we established an experimental condition including low stimulus intensity and short trial lengths (Ikeda et al., 2004). In our study, an intervening-stimulus paradigm was employed as the experimental procedure (Näätänen etal.,1988), i.e., participants were presented two kinds of tone pips (standard and probe tones) with the same probability to one ear. The other ear was masked by a white noise according to the standard ABR measurement. The auditory stimuli were delivered at a low intensity (40 dB SPL). The tasks for participants were either counting probes (attentive condition) or reading books (ignoring condition). Scalp potentials during the tasks were derived from electrodes placed at the vertex and an earlobe. The potentials were averaged over 500 trials and then compared between the attentive and ignoring conditions. By this, we found that the potentials within the 10-ms latency tended to demonstrate a general shift relative to the pre-stimulus baseline (Figure 2), as shown in the previous studies (Brix, 1984; Connolly et al., 1989; Hirschhorn \& Michie, 1990; Lukas, 1980, 1981; Picton et al., 1981; Woods \& Hillyard, 1978). Consistent with the studies, the direction of the potential shift failed to be stable in one polarity across participants. Thus, the information meaningful for discriminating the two conditions might be limited to the displacement of potentials itself. For representing the displacement of potentials appropriately, a measure (displacement percentage) was devised (See Methodological Principle). In a preliminary work using the measure, the task-related difference between the two conditions was evidently detected (Ikeda et al., 2004). The present article would provide further extensions and detailed descriptions of our study. 


\section{MATERIALS AND METHODS}

\section{Participants}

Twelve healthy-hearing and right-handed adults ( 8 women) participated in this study. Their mean age \pm SD was $32.2 \pm 10.0$ years. All participants showed hearing thresholds of $15 \mathrm{~dB}$ HL or less for both ears at conventional audiometric frequencies of 0.5 , $1,2,4$, and $8 \mathrm{kHz}$ that were behaviorallymeasured. Informed consent for the testing was obtained from all of them.

\section{Stimuli}

Identical to a previous study (Kodera et al., 1977), tone pips of 10-ms duration (5-ms ramps without plateau) were used. An intensity was $40 \mathrm{~dB}$ SPL. Stimuli were generated by a stimulator (Nihon Kohden SMP-4100) and then presented through a headphone (Elega DR-531) to the left ear by a stimulation rate of $3 \mathrm{~Hz}$. The polarity of stimuli was alternated for avoiding the evocation of cochlear microphonics or the FFR. The right ear continuously received a white noise of the same intensity.

\section{Procedure}

Participants received standard $(0.5 \mathrm{kHz}$ and probability 0.5$)$ and probe (either 1,2 , or $8 \mathrm{kHz}$ and probability 0.5 ) tones. The experimental conditions were either reading books (ignoring condition) or mentally counting the number of probes (attentive condition) during the stimulation. The order of probe tones was balanced across participants ina Latinsquaredesign. Theignoring condition was first conducted and then the attentive condition was performed on another day. To explain displacement measure of potentials and compare itwith other measures in detail, the present study focused on the results of a pair of $0.5-$ and 2-kHz tones.

\section{Physiological Recordings}


Scalp potentials were recorded by placing Ag/AgCl electrodes at the vertex (Cz) with reference to the left or right earlobes (A1 or A2). The ground electrode was attached to the forehead. Impedance between electrodes was less than $5 \mathrm{k} \Omega$. The potentials were amplified (gain $=20$ k) by an amplifier (Dia Medical system DPA-2008) with band pass of 16-3000 Hz (-6 and $-12 \mathrm{~dB} /$ octave for low- and high-cut filters, respectively). Using a personal computer with a 16-bit converter and a software (Kissei comtec EPLYZERII-A), the potentials were digitized at a sampling frequency of $5 \mathrm{kHz}$ and averaged over 500 epochs for each stimulus category. The analysis window was 63 ms including a 50-ms pre-stimulus baseline, and trials with a change exceeding $\pm 25 \mu \mathrm{V}$ were omitted from the averaging. An averaged potential derived from electrodes at the left earlobe and the vertex was denoted as an Al-potential, and that from the right earlobe and the vertex was as an A2-potential.

\section{Data Analysis}

In the present study, displacement of potentials within a period is defined as a deviation of the potentials from zero level. The concept of the displacement of potentials is illustrated in Figure 1. When lines $I_{1}$ and $I_{2}$ have the same length, the relationship between $m_{1}$ and $m_{2}$ (or $n_{1}$ and $n_{2}$ ) depends on a point 0 which divides $l_{1}$ or $l_{2}$ internally. In the figure, the relationship is $m_{1}<m_{2}$ when $m_{1} \geq n_{1}$ and $m_{2} \geq n_{2}$. If length of $l_{1}$ or $l_{2}$ and the point 0 represent the magnitude and zero level of potentials, respectively, the relationship between $m_{1}$ and $m_{2}$ indicates the displacement degree of potentials. Thus, the displacement of $l_{2}$ is greater than that of $l_{1}$.

This article concentrated on analyzing the displacement of potentials within a period after stimulus onset. Potentials were 
measured and evaluated for the standards $(0.5 \mathrm{kHz})$ and probes (2 $\mathrm{kHz})$, respectively. Three measures, that might represent the displacement of potentials, were then calculated and compared for each wave. First, maximum (or minimum) amplitude within a 13-ms post-stimulus period was determined. Second, a maximum (or minimum) among the next six means was derived from potentials in the post-stimulus period. Thus, the 13-ms epoch was divided into 6 intervals using modified Fibonacci sequence for weighting earlier time, i.e., $0<x \leq 1,1<x \leq 2,2<x \leq 3,3<x \leq 5,5<x \leq$ $8,8<x \leq 13$, and data points (a unit of $\mu V$ ) within each interval were averaged. The above intervals were introduced by considering the results in pilot studies, and their validity was also examined inalatersection of this article. For the first and secondmeasures, a maximum was either a positive or absolute value of potentials and a minimum was a negative one. Third, "displacement percentage" was computed from the six means identical to those for calculating the second measure. The methodology of the third measure is explained in the next section in detail.

Differences in a measure were examined by a repeated two-way analysis of variance (ANOVA), i.e., ignoring and attentive conditions $\times A 1$ and A2 electrodes. For post-hoc multiple comparisons, the Bonferroni method was employed.

\section{DISPLACEMENT PERCENTAGE}

\section{Methodological Principle}

Relative to the scheme in Figure 1, the methodological principle of displacement percentage can be explained. In this method, the sum of absolute values of the 6 means derived from the post-stimulus intervals corresponds to length of $l_{1}$ or $l_{2}$. However, the sum can be altered depending on the change of the means, and then it cannot 
be constant over different measurements. This problem would be resolved by converting absolute values of the means into percentages of those, giving rise to that the sum always shows $100 \%$. To identify displacement degrees that correspond to $m_{1}$ and $m_{2}$ in Figure 1 , raw values of the 6 means with positive or negative signs are put in order according to their values. In a mathematical expression, they are substituted for $M_{k}\left(1 \leq k \leq 6\right.$ and $\left.M_{k}<M_{k+1}\right)$ resulting in $M_{1}<M_{2}<M_{3}<M_{4}<M_{5}<M_{6}$. Keeping this order, absolute values of the means are converted into percentages of those. This procedure is conducted by the following formula where $1 \leq k \leq 6$.

$$
R_{k}=\frac{\left|M_{k}\right|}{\sum_{i=1}^{6}\left|M_{i}\right|} \times 100
$$

In consequence, a minimum among the percentages shows the nearest value to zero level, which approximates to the point 0 in Figure 1. In the order of percentages (i.e., $\boldsymbol{R}_{1}$ to $\boldsymbol{R}_{6}$ ) obtained from formula $(1), R_{n}$ is assumed as a minimum among the percentages. Then, displacement degrees of potentials can be expressed by the sum of either $R_{1}$ to $R_{n-1}$ or $R_{n+1}$ to $R_{6}$ (i.e., $S_{1}$ or $S_{2}$ ).

$$
\begin{aligned}
& S_{1}=\sum_{k=1}^{n-1} R_{k} \\
& S_{2}=\sum_{k=n+1}^{6} R_{k}
\end{aligned}
$$

Displacement percentage $(D)$ is finally given by either $S_{1}$ or $S_{2}$ that is greater than the other.

\section{Application to Single Case}

To clarify the procedure for calculating displacement percentages, the transformation of raw data in a participant was illustrated in Figures 2 and 3. The participant was a woman aged 19.2 years 
old. Figures 2a-d show averaged waveforms and corresponding " $D$ " values for each stimulus category in both conditions, obtained from the Al-potential. The magnitude of $D$ tended to increase in the attentive condition compared with the ignoring condition, particularly for $2-\mathrm{kHz}$ tone. Such a tendency in $D$ seems to be achieved by a general shift of potentials from baseline within the latency of $8 \mathrm{~ms}$, but not by peaks of specific components. Although the direction of the displacement in this case was negative, the opposite direction was also found among participants. Figures $2 e$ and $2 f$ exhibit an example of positive shift observed in a woman aged 19.4 years old.

Figure 3 demonstrates the process of calculating $D$ by using 6 means obtained from potentials for the probes in the ignoring condition (Figures $2 \mathrm{c}$ and $3 \mathrm{a}$ ). The means corresponding to each post-stimulus interval are shown in Figure 3a. Because the numbers at four places or after of decimals were abbreviated for each mean in Figure 3a, the value of $D$ in Figure 3b disagrees with that in Figure 2c. From steps 1 to 2 in Figure 3b, the means are sorted according to their values. Maintaining the order, they are converted into absolute values at step 3. The sum of them is 0.186 . At step 4, percentages of each absolute value are computed using the sum (0.186). Because the minimum among them is $1.1 \%, S_{1}$ and $S_{2}$ become 65 and $33.9 \%$, respectively. In consequence, $D$ is $65 \%$ since $S_{1}>S_{2}$.

\section{COMPARISON OF DIFFERENT MEASURES}

\section{Maximum (Minimum) Amplitude within the Post-Stimulus Period}

Table 1 displays the results of ANOVA for maximum or minimum amplitudes within the 13-ms period. None of significant effects was found in the table. Only a main effect of electrode for the 
0.5-kHz tone approached to a significant level in negative amplitudes .

\section{The Maximum (Minimum) among Six Means in the Post-Stimulus Intervals}

The results of ANOVA for the maximum or minimum value among the means, computed from the 6 intervals, are shown in Table 2. Any main effect was statistically insignificant, although a condition effect for the $2-\mathrm{kHz}$ tone approached to significance in positive amplitudes. An interaction between factors of condition and electrode was significant for the 0.5-kHz tone in negative polarity. However, the multiple comparisons revealed no significant difference $(p s>.06)$.

\section{Displacement Percentage}

Table 3 demonstrates the ANOVA results for displacement percentages. Interaction plots between factors of condition and electrode are illustrated in Figure 4. Contrary to the two measures already described, a highly significant main effect of condition was detected in the $2-\mathrm{kHz}$ tone, indicating that $D$ was greater during the attentive condition than during the ignoring condition (Figure 4b). In addition, displacement percentages for the other pairs of stimuli (the standards and the probes of either 1 or $8 \mathrm{kHz}$ ) were evaluated. As shown in Table 3, any $D$ value in those cases failed to reach a significant level.

\section{Examination of Intervals for Computing Means}

For examining the validity of the 6 intervals to derive means from potentials, three alternative methods for determining intervals were tested. First, the 12-ms post-stimulus epoch was divided by each $2 \mathrm{~ms}$ into 6 intervals; second, the first half of the 12-ms period was segmented by 1-ms intervals; third, the second half of the same periodwas divided into 1-ms segments. Sixmeans derived 
from each method were converted into $D$ values. Although the original method using modified Fibonacci sequence yielded a significant main effect of condition for the $2-\mathrm{kHz}$ tone $(p<.01)$, the alternatives applied to the same data showed no significance for the condition effect, i.e., the first method, $F(1,11)=.66$, $p=.44$; the second method, $F(1,11)=4.02, p=.07$; the third method, $F(1,11)=.27, p=.61$. A relatively high $F$ value in the second method may suggest that earlier time in the post-stimulus period might be critical for detecting attention effects. These results would support the validity of the weighted intervals that were used for computing means.

\section{DISCUSSION}

In the present results, the task-related difference between the ignoring and attentive conditions was possible to be identified by a measure in itself reflecting the displacement of potentials within a period (i.e., displacement percentage) as shown in Table 3. If using either raw or mean amplitudes within the same period, however, the condition-related effects in the ANOVA or post-hoc comparisons failed to achieve statistical significances for any polarity (Tables 1 and 2). Thus, this might corroborate the observation that the early displacement of potentials was not specific to one polarity relative to the baseline (Brix, 1984; Connolly et al., 1989; Hirschhorn \& Michie, 1990; Ikeda et al., 2004; Lukas, 1980, 1981; Picton et al., 1981; Woods \& Hillyard, 1978). In the following considerations, we endeavor to refuse the possibility that the task-related displacement could be assigned to artifacts other than neural events in the auditory pathway.

As already discussed in previous studies using the OAE (Giard et al., 1994; Puel et al., 1988, 1989), the middle ear muscle reflex 
is a factor which could influence measures of auditory peripheral functions. However, the reflex would be implausible for contributing the present results employing low stimulus intensity, because it would be elicited at tonal intensity beyond 80-90 dB SL in humans (McPherson \& Thompson, 1978). The second risk factor would be the background electrical noise such as scalp musculature activity and eye or body movements. Nevertheless, it seems difficult to consider that an increased $D$ in the attentive condition could be attributed to the background noise, since the threshold of artifact rejection in the present study $( \pm 25 \mu \mathrm{V})$ rarely omitted trials (less than 10 trials) in both conditions for any participant. Even though the rejection standard was set more strict $( \pm 15 \mu \mathrm{V})$, the above results were kept for 10 participants. Moreover, if the background noise greatly contributed to the task-related difference between the conditions, a high significance of statistics would be obtained not only from 2-kHz probes but also from standards and other probes (Table 3). In fact, the significant outcome was identified only for 2-kHz probes, whichmight contradict with a great contribution of the background noise to the effect. It is unknown why the task-related difference was found only for 2-kHz tones, whereas in a previous study where the right ear was stimulated with tone pips, we obtained data that the task-related effect was difficult to occur for 8-kHz tones (Ikeda et al., 2004). This seems to be consistent with the studies showing that higher frequency tones ( $5 \mathrm{kHz}<$ ) were inappropriate for establishing the peripheral attention effect (Lukas, 1980; Oatman \& Anderson, 1977). A remaining problem to eliminate possible artifacts may be a relatively short number of averaging (500 trials), although it was adopted by considering Lukas's $(1980,1981)$ recommendation 
for using short trial lengths as an experimental condition. We obtained in the previous study (Ikeda et al., 2004) an opportunity for testing the influence of the number of averaged epochs on $D$ values. Thus, seven participants could receive 1000 trials for each stimulus category, and then two conditions of averaging, i.e., 500 and 1000 epochs, were compared for examining the influences on statistics. In general impression, the increased averaging appeared to improve statistical significances. For instance, a task-related effect showing tendency to significance in the 500 -epoch condition, $F(1,6)=4.80, p=.07$, gave rise to reaching a significant level by averaging 1000 epochs, $F(1,6)=6.53, p$ $=.04$. From this, it might be inferred that a statistical significance detected in the 500-epoch condition can survive even though the averaged trials are more increased. Considering the above factors that could yield various artifacts, there seems much justice in the argument that the task-dependent displacement of potentials can be a phenomenon based on neural events in the auditory peripheral pathway, and they are bidirectional in polarity due to neither myogenic activities nor experimental artifacts.

As an alternative to the idea that the above potential shift is based on neural events, there is a possibility that it may be a phenomenon occurring within the cochlea. A candidate corresponding to the idea might be the summating potential (SP) that can be recorded as one component of electrocochleography. Since the SP is an evoked change of DC component after stimulus onset and is revealed as a positive or negative displacement of DC during stimulation (Dallos et al., 1972), it is possible to build up an assumption that the present measure has identified the deteriorated SP from scalp potentials. However, the ANOVA for 
displacement percentages revealed a significant effect of condition but not a significant interaction with electrodes (Table 3). This suggests that the attention effect was derived from electrodes placed at both earlobes (Figure 4), although tone pips were exposed only to the left ear and the opposite ear was masked by a white noise. Because the attention effect was not limited to the stimulated side, it seems difficult to validate an argument that the potential displacement in the attentive condition is a phenomenon localized within the cochlea (Picton et al., 1981). In contrast, the ABR can be recorded not only from an electrode of the stimulated side but also from the one of the masked side (Reid \& Thornton, 1983). Therefore, an appropriate idea may be rather that the potential displacement can be neural events originated from a portion at least outside the cochlea, e.g., the auditory brainstempathway. At the same time, we have an impression that the FFR is unrelated to the potential shift found in the attentive condition, though the wave is an ERP demonstrating positive results for the peripheral attention effect (Galbraith et al., 1998; Hoormann et al., 2000). This is because the present procedure employed alternating stimulus polarity to refute the contribution of the FFR, and the component ordinarily appears following wave $V$ of the ABR (Picton et al., 1981).

Based on filtering out particular bandwidths, the ABR can be divided into two components, i.e., slow and fast components with frequency ranges of $20-300 \mathrm{~Hz}$ and 400-3000 $\mathrm{Hz}$, respectively (Ehara et al., 1983; Suzuki et al., 1982, 1986). Fast components correspond to a series of positive waves such as waves I-V, while a slow component represents a positive deflection having the peak latency approximately consistent with wave V. Among the two 
components, the slow component seems to be a candidate that might fit the early potential displacement observed in this study. Power spectra corresponding to the slow component show robustness for decreased intensity, although those corresponding to the fast components markedly decrease in accordance with intensity (Suzuki et al., 1982). This property of the slow component might be favorable for explaining the present outcomes that the task-related displacement appeared at a low intensity (40 dB SPL). Moreover, even though the slow component reveals a prominent positive peak, its potentials up to the positive peak can fluctuate in both polarities depending on exogenous and endogenous parameters (Ehara etal., 1983; Suzuki etal.,1986). In particular, vigilance levels have a major effect on the slow component, i.e., the fluctuation of the component increases during sleep compared to wakefulness, whereas the fast components are almost independent of vigilance levels (Ehara et al., 1983). The susceptibility of waveforms due to vigilance levels might be analogous to cortical ERPs whose waveforms show alterations during sleep relative to wakefulness (Williams et al., 1962). It is considered that the peripheral attention effect can be established depending on the auditory efferent pathway which emerges from the auditory cortex and, through the superior olivary complex, arrives at outer hair cells in the cochlea (Lukas, 1980, 1981; Puel et al., 1988, 1989; Warr \& Guinan, 1979) . Considering a linkage between the facts, it may be assumed that the slow component of ABR can be easily affected by the auditory efferent pathway, and then central factors such as attention and arousal can resultin alterations of the component. At the present, thus, the early potential displacement found in this study may be justified by behaviors of the slow component of ABR. 


\section{References}

Bauer, L. O., \& Bayles, R. L. (1990). Precortical filtering and selective attention: An evoked potential analysis. Biological Psychology, 30, 21-33.

Brix, R. (1984). The influence of attention on the auditory brain stem evoked responses. Acta Otolaryngologica, 98, 89-92.

Connolly, J. F., Aubry, K., McGillivary, N., \& Scott, D.W. (1989). Human brainstem auditory evoked potentials fail to provide evidence of efferent modulation of auditory input during attentional tasks. Psychophysiology, 26, 292-303.

Dallos, P., Schoeny, Z. G., \& Cheatham, M. A. (1972). Cochlear summating potentials: Descriptive aspects. Acta otolaryngologica, Supplement 302, 1-46.

Davis, A. E., \& Beagley, H. A. (1985). Acoustic brainstem responses for clinical use: The effect of attention. Clinical Otolaryngology, 10, 311-314.

Ehara, Y., Kawamura, S., Ichikawa, G., Harada, K., Yoshikawa, H., \& Ono, I. (1983). Influence of vigilance levels on slow and fast components of auditory brainstem responses. Audiology Japan, 26, 493-494. (In Japanese)

Ferber-Viart, C., Duclaux, R., Collet, L., \& Guyonnard, F. (1995). Influence of auditory stimulation and visual attention on otoacoustic emissions. Physiology and Behavior, 57, 1075-1079. Froehlich, P., Collet, L., \& Morgon, A. (1993). Transiently evoked otoacoustic emission amplitudes change with changes of directed attention. Physiology and Behavior, 53, 679-682.

Galbraith, G. C., Bhuta, S. M., Choate, A. K., Kitahara, J. M., \& Mullen, T.A. (1998). Brain stem frequency-following response to dichotic vowels during attention. Neuroreport, 9, 1889-1893. 
Giard, M.-H., Collet, L., Bouchet, P., \& Pernier, J. (1994). Auditory selective attention in the human cochlea. Brain Research, 633, $353-356$.

Gregory, S. D., Heath, J. A., \& Rosenberg, M. E. (1989). Does selective attention influence the brain-stem auditory evoked potential? Electroencephalography and Clinical

Neurophysiology, 73, 557-560.

Hackley, S.A., Woldorff, M., \&Hillyard, S.A. (1990). Cross-modal selective attention effects on retinal, myogenic, brainstem, and cerebral evoked potentials. Psychophysiology, 27, 195-208. Hernández-Peón, R., Scherrer, H., \& Jouvet, M. (1956). Modification of electric activity in cochlear nucleus during "attention" in unanesthetized cats. Science, 123, 331-332.

Hirschhorn, T. N., \& Michie, P. T. (1990). Brainstem auditory evoked potentials (BAEPS) and selective attention revisited.

Psychophysiology, 27, 495-512.

Hoormann, J., Falkenstein, M., \& Hohnsbein, J. (2000). Early attention effects in human auditory-evoked potentials. Psychophysiology, 37, 29-42.

Ikeda, K., Hayashi, A., Sekiguchi, T., \& Era, S. (2004). Auditory attention at periphery detected from human scalp potentials. International Journal of Psychology, 39(5-6), 29. Kodera, K., Yamane, H., Yamada, O., \& Suzuki, J-I. (1977). Brain stem response audiometry at speech frequencies. Audiology, 16, $469-479$.

Kuk, F. K., \& Abbas, P. J. (1989). Effects of attention on the auditory evoked potentials recorded from the vertex (ABR) and the promontory (CAP) of human listeners. Neuropsychologia, 27, $665-673$. 
Lukas, J. H. (1980). Human auditory attention: The olivocochlear bundle may function as a peripheral filter. Psychophysiology, 17, $444-452$.

Lukas, J. H. (1981). The role of efferent inhibition in human auditory attention: An examination of the auditory brainstem potentials. International Journal of Neuroscience, 12, $137-145$.

Marsh, J. T., Worden, F. G., \& Hicks, L. (1962). Some effects of room acoustics on evoked auditory potentials. Science, 137, $280-282$.

McCallum, W. C., Curry, S. H., Cooper, R., Pocock, P. V., \& Papakostopoulos, D. (1983). Brain event-related potentials as indicators of early selective processes in auditory target localization. Psychophysiology, 20, 1-17.

McPherson, D. L., \& Thompson, D. (1978). Quantification of the threshold and latency parameters of the acoustic reflex in humans. Acta Otolaryngologica, Supplement 353, 1-37. Meric, C., \& Collet, L. (1992). Visual attention and evoked otoacoustic emissions: A slight but real effect. International Journal of Psychophysiology, 12, 233-235.

Näätänen, R., Sams, M., Alho, K., Paavilainen, P., Reinikainen, K., \& Sokolov, E. N. (1988). Frequency and location specificity of the human vertex N1 wave. Electroencephalography and Clinical Neurophysiology, 69, 523-31.

Oatman, L. C. (1971). Role of visual attention on auditory evoked potentials in unanesthetized cats. Experimental Neurology, 32, $341-356$.

Oatman, L. C. (1976). Effects of visual attention on the intensity of auditory evoked potentials. Experimental Neurology, 51, 
$41-53$.

Oatman, L.C., \& Anderson, B.W. (1977). Effects of visual attention on tone burst evoked auditory potentials. Experimental Neurology, 57, 200-211.

Picton, T. W., \& Hillyard, S. A. (1974). Human auditory evoked potentials. II: Effects of attention. Electroencephalography and Clinical Neurophysiology, 36, 191-199.

Picton, T. W., Hillyard, S. A., Galambos, R., \& Schiff, M. (1971). Human auditory attention: A central or peripheral process? Science, 173, 351-353.

Picton, T.W., Stapells, D. R., \& Campbell, K. B. (1981). Auditory evoked potentials from the human cochlea and brainstem. Journal of Otolaryngology, 10 supplement 9, 1-41.

Puel, J.-L., Bonfils, P., \& Pujol, R. (1988). Selective attention modifies the active micromechanical properties of the cochlea. Brain Research, 447, 380-383.

Puel, J.-L., Rebillard, G., Bonfils, P., \& Pujol, R. (1989). Effect of visual selective attention on otoacoustic emissions. In J. P. Wilson \& D. T. Kemp (Eds.), Cochlear mechanisms. Structure, function and models (pp. 315-321). New York: Plenum Press. Reid, A., \& Thornton, A. R. D. (1983). The effects of contralateral masking upon brainstem electric responses. British Journal of Audiology, 17, 155-162.

Suzuki, T., Kobayashi, K., \& Takagi, N. (1986). Effects of stimulus repetition rate on slow and fast components of auditory brain-stem responses. Electroencephalography and Clinical Neurophysiology, 65, 150-156.

Suzuki, T., Sakabe, N., \& Miyashita, Y. (1982). Power spectral analysis of auditory brain stem responses to pure tone stimuli. 
Scandinavian Audiology, 11, 25-30.

Warr, W. B., \& Guinan, J. J. Jr. (1979). Efferent innervation of the organ of corti: Two separate systems. Brain Research, 173, $152-155$

Wickelgren, W. O. (1968). Effects of walking and flash stimulation on click-evoked responses in cats. Journal of Neurophysiology, $31,769-776$.

Williams, H. L., Tepas, D. I., \& Morlock, H. C. (1962). Evoked responses to clicks and electroencephalographic stages of sleep in man. Science, 138, 685-686.

Woldorff, M. G., \& Hillyard, S. A. (1991). Modulation of early auditory processing during selective listening to rapidly

presented tones. Electroencephalography and Clinical

Neurophysiology, 79, 170-191.

Woods, D. L., \& Hillyard, S. A. (1978). In D. A. Otto (Ed.), Multidisciplinary perspectives in event-related brain potential research (pp. 230-233). Washington, D.C.: U.S. Government Printing Office. 
Author Note

We thank our colleagues, in particular Tatsuya Yaguchi and Akikazu Katoh for theirgenerous assistance. We are also indebted to Profs. Souichi Hashimoto and Toshisada Deguchi for their helpful suggestions in the course of the experiments.

Address correspondence to Dr. Kazunari Ikeda, Laboratory of Cognitive Psychophysiology, Faculty of Education, Tokyo Gakugei University, Koganei, Tokyo 184-8501, Japan. E-mail: kazunarieu-gakugei.ac.jp 
TABLE 1. Results obtained from a repeated two-way ANOVA for maximum or minimum amplitudes within the post-stimulus period (13 ms)

\begin{tabular}{|c|c|c|c|c|c|}
\hline Wave & Polarity & Effect & $d f$ & $F$ & $p$ \\
\hline \multirow[t]{9}{*}{$0.5 \mathrm{kHz}$} & Positive & Condition & $1 / 11$ & 0.35 & 0.57 \\
\hline & & Electrode & $1 / 11$ & 0.37 & 0.55 \\
\hline & & Interaction & $1 / 11$ & 2.26 & 0.16 \\
\hline & Negative & Condition & $1 / 11$ & 0.00 & 0.97 \\
\hline & & Electrode & $1 / 11$ & $3.85 \dagger$ & 0.08 \\
\hline & & Interaction & $1 / 11$ & 0.21 & 0.66 \\
\hline & Absolute & Condition & $1 / 11$ & 0.01 & 0.93 \\
\hline & & Electrode & $1 / 11$ & 1.01 & 0.34 \\
\hline & & Interaction & $1 / 11$ & 0.71 & 0.42 \\
\hline \multirow[t]{9}{*}{$2 \mathrm{kHz}$} & Positive & Condition & $1 / 11$ & 0.74 & 0.41 \\
\hline & & Electrode & $1 / 11$ & 0.01 & 0.91 \\
\hline & & Interaction & $1 / 11$ & 0.00 & 0.97 \\
\hline & Negative & Condition & $1 / 11$ & 0.01 & 0.92 \\
\hline & & Electrode & $1 / 11$ & 1.81 & 0.21 \\
\hline & & Interaction & $1 / 11$ & 0.23 & 0.64 \\
\hline & Absolute & Condition & $1 / 11$ & 0.00 & 0.98 \\
\hline & & Electrode & $1 / 11$ & 0.06 & 0.81 \\
\hline & & Interaction & $1 / 11$ & 0.53 & 0.48 \\
\hline
\end{tabular}


TABLE 2. Results of a repeated two-way ANOVA for the maximum or minimum among six means derived from the post-stimulus intervals

\begin{tabular}{|c|c|c|c|c|c|}
\hline Wave & Polarity & Effect & $d f$ & $F$ & $p$ \\
\hline \multirow[t]{9}{*}{$0.5 \mathrm{kHz}$} & Positive & Condition & $1 / 11$ & 0.02 & 0.88 \\
\hline & & Electrode & $1 / 11$ & 1.95 & 0.19 \\
\hline & & Interaction & $1 / 11$ & 1.40 & 0.26 \\
\hline & Negative & Condition & $1 / 11$ & 0.56 & 0.47 \\
\hline & & Electrode & $1 / 11$ & 0.19 & 0.68 \\
\hline & & Interaction & $1 / 11$ & $7.48 *$ & 0.02 \\
\hline & Absolute & Condition & $1 / 11$ & 0.35 & 0.57 \\
\hline & & Electrode & $1 / 11$ & 3.07 & 0.11 \\
\hline & & Interaction & $1 / 11$ & 2.93 & 0.12 \\
\hline \multirow[t]{9}{*}{$2 \mathrm{kHz}$} & Positive & Condition & $1 / 11$ & $3.67+$ & 0.08 \\
\hline & & Electrode & $1 / 11$ & 0.10 & 0.76 \\
\hline & & Interaction & $1 / 11$ & 0.07 & 0.79 \\
\hline & Negative & Condition & $1 / 11$ & 0.12 & 0.74 \\
\hline & & Electrode & $1 / 11$ & 1.36 & 0.27 \\
\hline & & Interaction & $1 / 11$ & 0.59 & 0.46 \\
\hline & Absolute & Condition & $1 / 11$ & 0.08 & 0.78 \\
\hline & & Electrode & $1 / 11$ & 0.02 & 0.88 \\
\hline & & Interaction & $1 / 11$ & 0.03 & 0.86 \\
\hline
\end{tabular}

$+p<.10 ; \star p<.05$. 
TABLE 3. Results of a repeated two-way ANOVA for displacement percentages

\begin{tabular}{|c|c|c|c|c|}
\hline Wave & Effect & $d f$ & $F$ & $p$ \\
\hline \multirow{3}{*}{ for $1 \mathrm{kHz}$} & Condition & $1 / 11$ & $3.63+$ & 0.08 \\
\hline & Electrode & $1 / 11$ & 0.54 & 0.48 \\
\hline & Interaction & $1 / 11$ & 0.00 & 0.99 \\
\hline \multirow[t]{3}{*}{$1 \mathrm{kHz}$} & Condition & $1 / 11$ & 0.10 & 0.76 \\
\hline & Electrode & $1 / 11$ & 0.29 & 0.60 \\
\hline & Interaction & $1 / 11$ & 0.01 & 0.94 \\
\hline $0.5 \mathrm{kHz}$ & Condition & $1 / 11$ & 0.06 & 0.81 \\
\hline \multirow[t]{2}{*}{ for $2 \mathrm{kHz}$} & Electrode & $1 / 11$ & 1.01 & 0.34 \\
\hline & Interaction & $1 / 11$ & 2.81 & 0.12 \\
\hline \multirow[t]{3}{*}{$2 \mathrm{kHz}$} & Condition & $1 / 11$ & $11.89 * *$ & 0.01 \\
\hline & Electrode & $1 / 11$ & 1.42 & 0.26 \\
\hline & Interaction & $1 / 11$ & 0.12 & 0.74 \\
\hline $0.5 \mathrm{kHz}$ & Condition & $1 / 11$ & 0.68 & 0.43 \\
\hline \multirow[t]{2}{*}{ for $8 \mathrm{kHz}$} & Electrode & $1 / 11$ & 1.85 & 0.20 \\
\hline & Interaction & $1 / 11$ & 0.77 & 0.40 \\
\hline \multirow[t]{3}{*}{$8 \mathrm{kHz}$} & Condition & $1 / 11$ & 2.24 & 0.16 \\
\hline & Electrode & $1 / 11$ & $3.59+$ & 0.09 \\
\hline & Interaction & $1 / 11$ & 0.05 & 0.83 \\
\hline
\end{tabular}


Figure Captions

FIGURE 1. A diagram explaining the concept of displacement in the present study. In this, $l$ ines $l_{1}$ and $l_{2}$ stand for the same magnitude. The point 0 represents a common standard such as zero level. If the point 0 divides $I_{1}$ and $l_{2}$ internally, and the relation of interior divisions is $m_{1} \geq n_{1}$ and $m_{2} \geq n_{2}$, displacement of $m_{2}$ is then greater than that of $m_{1}$.

FIGURE 2. Average waveforms derived from electrodes placed at the vertex (Cz) and a left earlobe (A1) under the ignoring (left) and attentive (right) conditions. (a-d) Waves of a woman aged 19.2 years old: ( $a$, b) "Waves for Standards $(0.5-\mathrm{kHz}$ Tones)" and (c, d) "Those for Probes (2-kHz Tones)". (e, f) Waves for probes recorded from a woman aged 19.4 years old. The upper deflection is positive. The $D$ value shows displacement percentage in each wave.

FIGURE 3. Graphical representations of procedures for calculating displacement percentage. (a) Six means each of which is derived from one of six post-stimulus intervals. The values are corresponding to those for probes in the ignoring condition (Figure 2c). (b) Steps for converting the initial means into the $D$ value. In step (2), the initial means in step (1) are put in order depending on their values. They are converted into absolute values in step (3), and further into percentages of those in step (4), e.g., $0.057 \div 0.186 \times 100=30.6$. In that order, the percentages either before or after the minimum $(=1.1 \%)$ are added. Among the resultant 
two sums (i.e., 65 and 33.9\%), the $D$ value is given by one greater than the other.

FIGURE 4. Mean displacement percentages averaged over participants $(N=12)$ as a function of electrode placement (A1 and A2): (a) "Standards" and (b) "Probes". Open circle "Ignoring Condition", closed circle "Attentive Condition". Vertical bars represent standard errors of mean. 


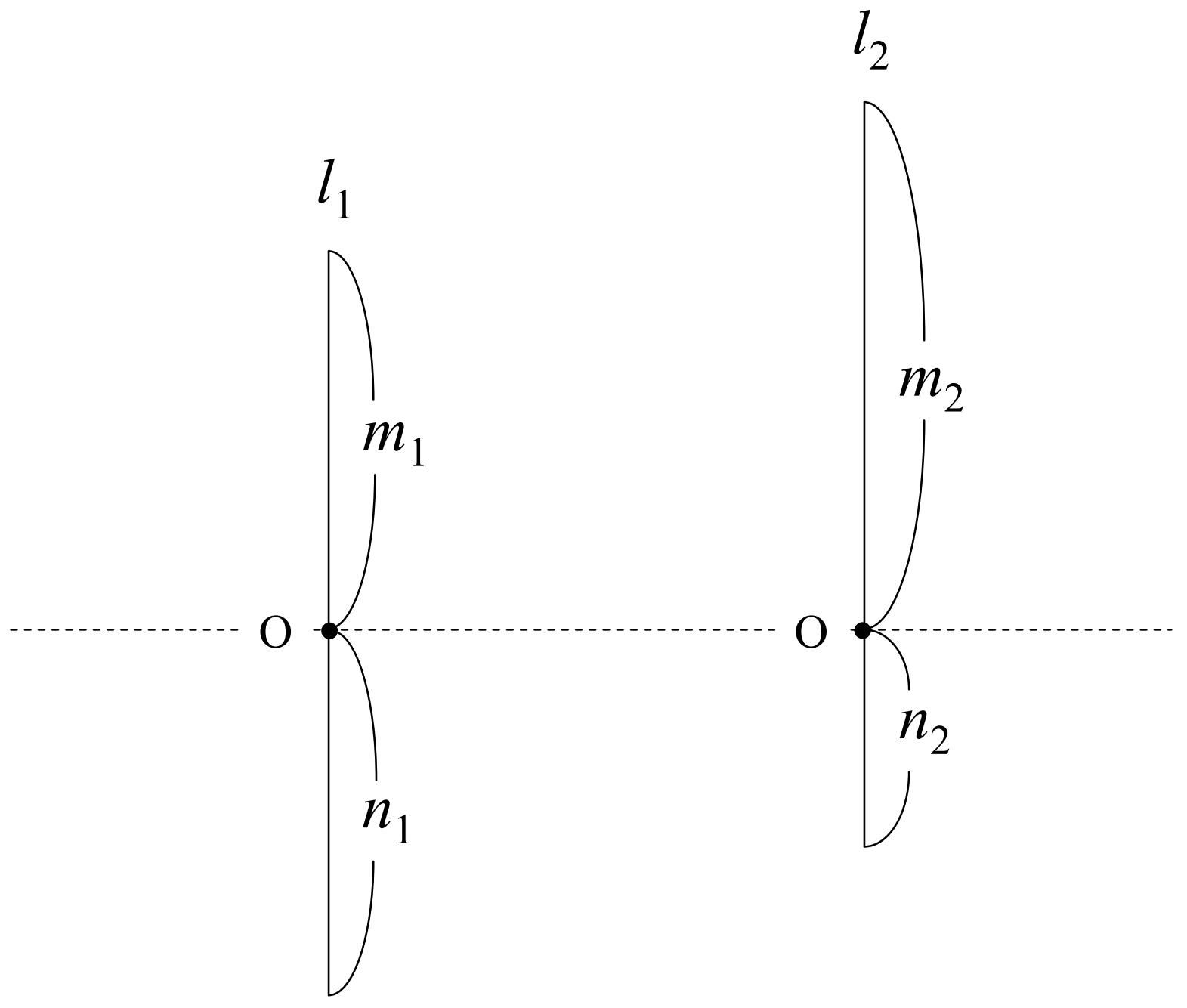


lgnoring
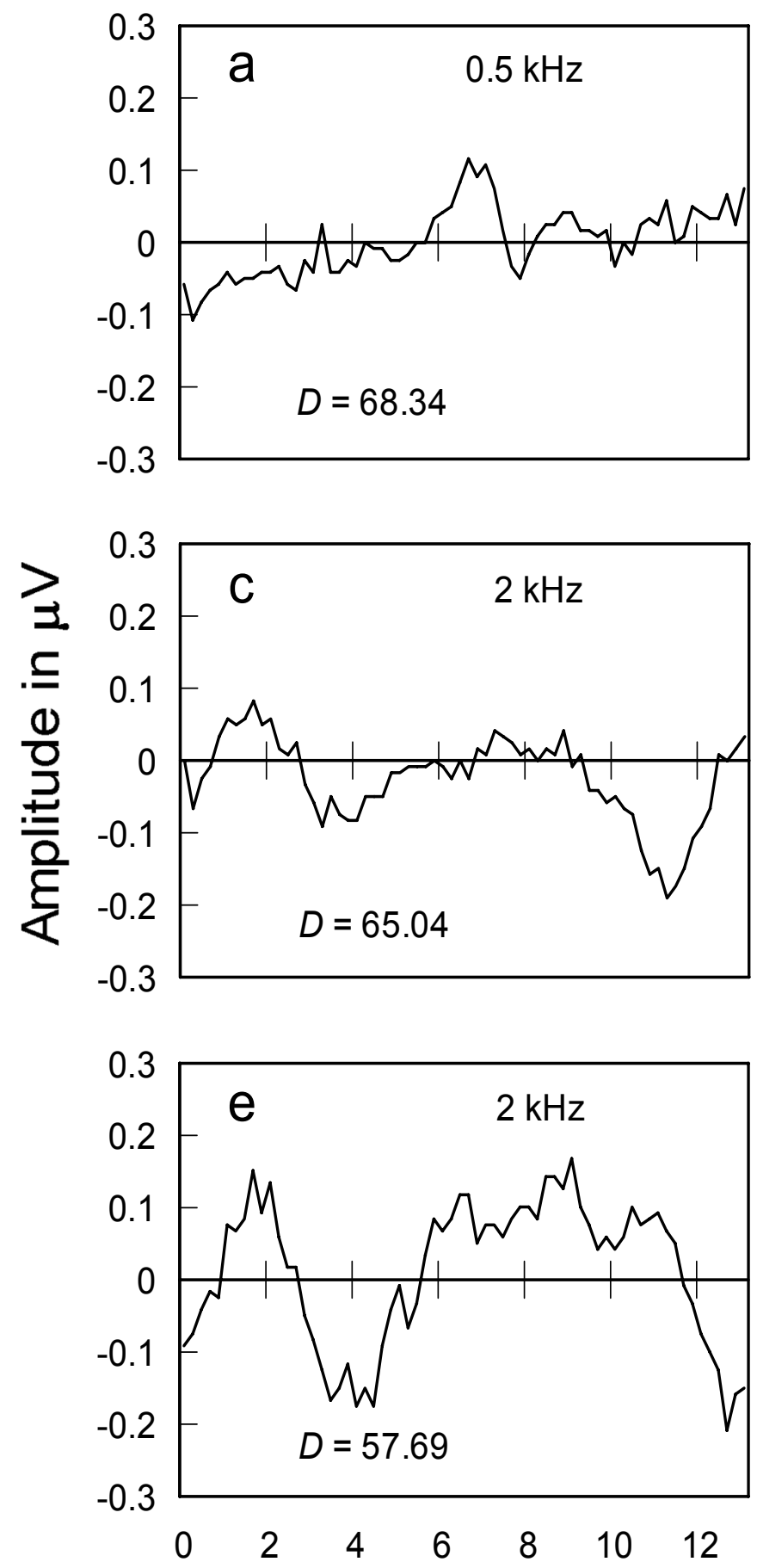

Attentive
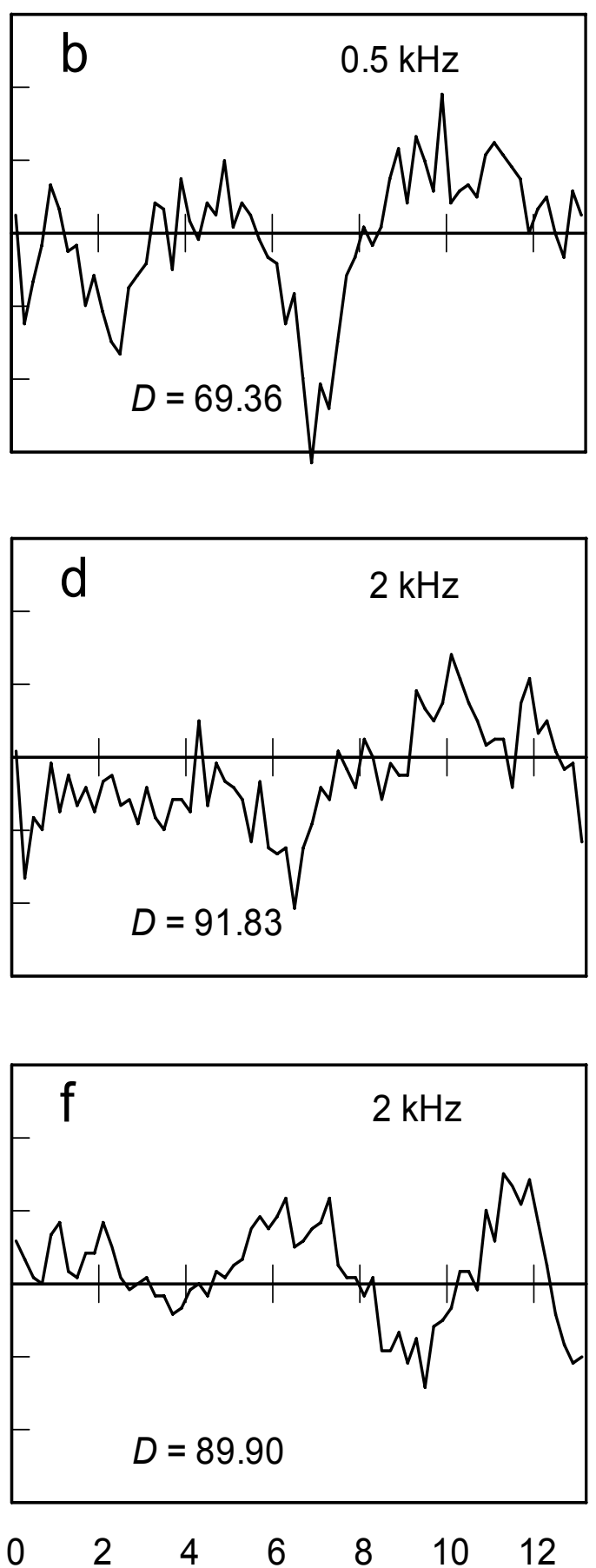

Post-stimulus time in ms 
a

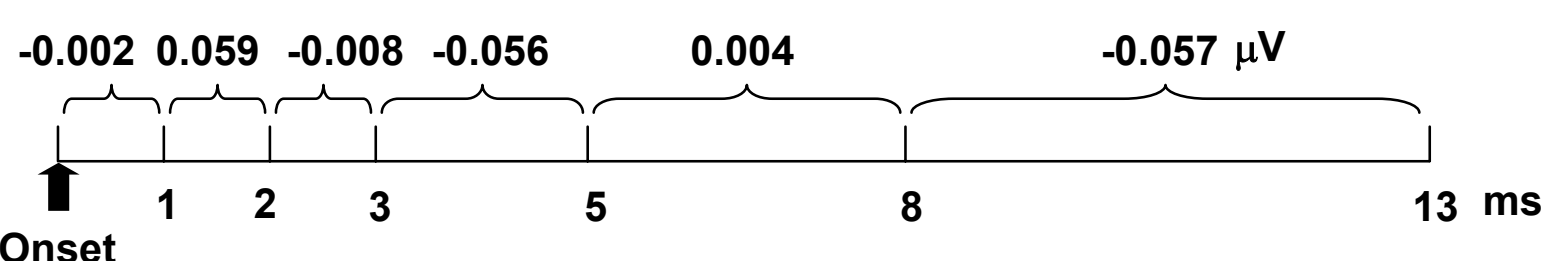

Onset

b

(1) $\quad \begin{array}{llllll}-0.002 & 0.059 & -0.008 & -0.056 & 0.004 & -0.057\end{array}$

1

(2) $\quad \begin{array}{rrrrrr}-0.057 & -0.056 & -0.008 & -0.002 & 0.004 & 0.059\end{array}$

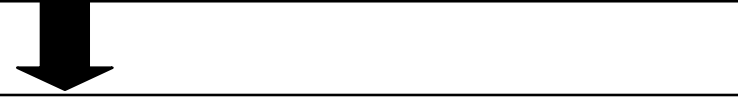

Sum

(3) $\begin{array}{lllllll}0.057 & 0.056 & 0.008 & 0.002 & 0.004 & 0.059 & 0.186\end{array}$

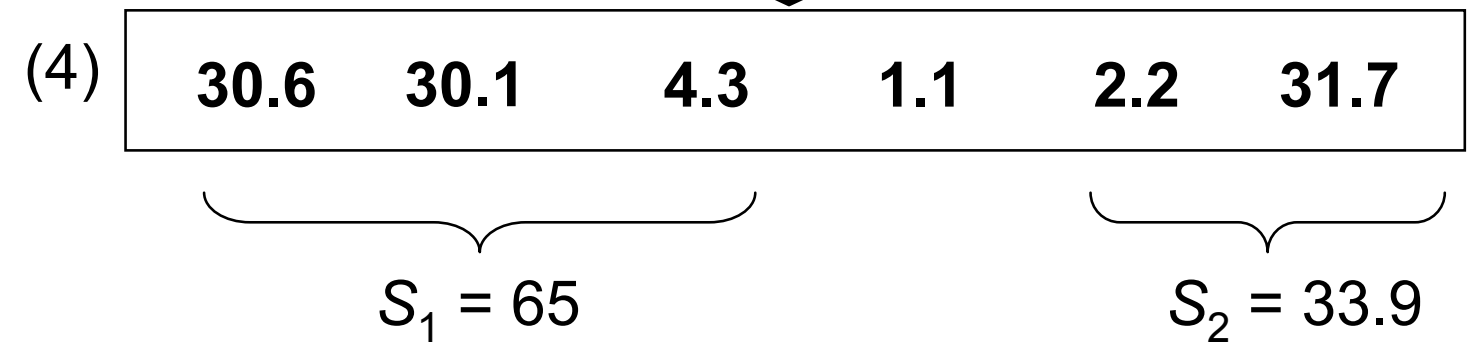

$D=65 \quad \because S_{1}>S_{2}$ 


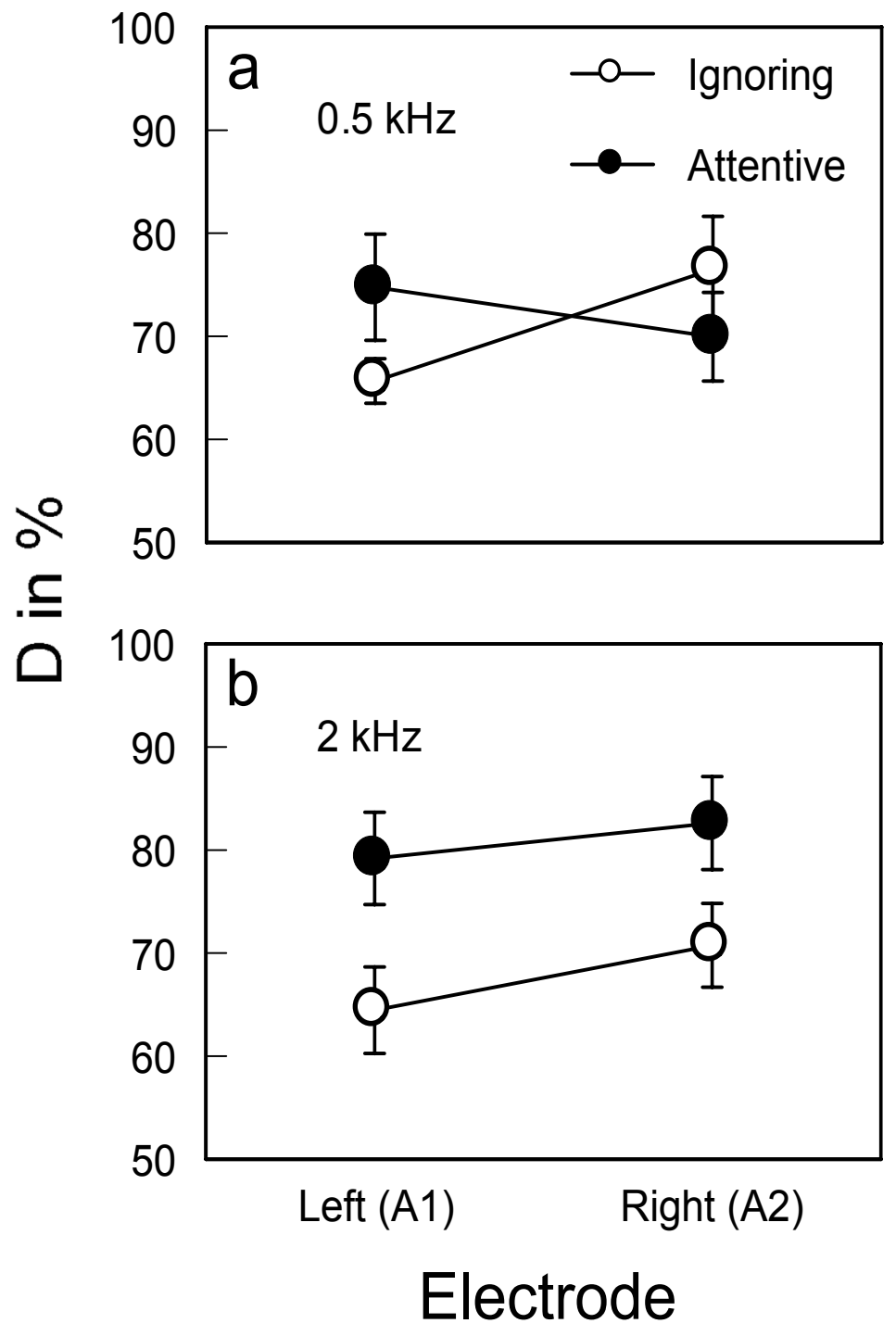

